\title{
EPISTEMOLOGÍA DE LA FORMACIÓN DOCENTE: UNA MIRADA CRÍTICA
}

\section{RESUMEN}

\author{
Pastor Umanzor \\ Departamento de Matemáticas \\ Centro Regional San Pedro Sula \\ Universidad Pedagógica nacional Francisco Morazán \\ pastoru2001@yahoo.com
}

Este ensayo sugiere una revisión de conceptos con origen universal y que se utilizan en la formación de profesionales de la educación de manera indistinta, lo que origina confusiones a la hora de diferenciar la formación de docentes de la formación docente. Igual sucede con los conceptos: métodos, técnicas, estrategias, procedimientos y procesos. Por experiencia propia y en conversaciones con diferentes profesionales en distintas universidades, profesores y estudiantes los aceptan como conceptos conocidos, aunque con diferentes significados.

De lo que trata el ensayo es intentar diferenciar esos conceptos y dar una perspectiva académica a la formación docente, que debe ser el resultado del intercambio de conocimientos sustantivos y sintácticos de las ciencias particulares y, principalmente, de los conocimientos pedagógicodidácticos-psicológicos atinentes al hecho formador: un profesor debe saber enseñar.

\section{PALABRAS CLAVE}

Epistemología de la educación, de la investigación, de la formación de docentes, de la formación docente.

\section{ABSTRACT}

This essay suggests a review of concepts with a universal origin and that are used in training education professionals interchangeably, resulting in confusion differentiating the training of teachers with teacher training (the preparation that a person possesses). The same happens with the concepts: methods, techniques, strategies, procedures and processes. By own experience and in discussions with different professionals in different universities, teachers and students accept them as known concepts, but with different meanings.

What this essay is about is an attempt of trying to differentiate these concepts and give an academic perspective to teacher training, which should be the result of the exchange of substantive and syntactic knowledge of sciences, and especially the pedagogical-didactic-psychological knowledge pertaining to the trainer issue: a teacher must know how to teach. 
Recibido 5 de abril de 2016 /Aceptado 6 de noviembre de2016

\section{KEY WORDS}

Epistemology of education, research, teacher education, teacher training.

\section{INTRODUCCIÓN}

Antes y ahora, la formación docente está en espera porque las actuales universidades están concentradas en formar un profesional de la abogacía, de la medicina o de otras especialidades, incluso de la educación con los mismos argumentos curriculares. Existen universidades pedagógicas que tienen como propósito la formación de docentes, pero no la formación docente.

Ambos tipos de universidades siguen fundamentos y expresiones de desarrollo académico: diseño y desarrollo de un currículo con un sistema de capacitación docente y un sistema de evaluación curricular, pero faltan mecanismos que controlen que la concreción del currículo se haga en la línea de lo diseñado. Incluso, estas universidades aceptan el nuevo discurso de la pedagogía crítica, pero a la hora de aplicar sus fundamentos y componentes, asumen y desarrollan acciones académicas propias del profesor, no las planificadas institucionalmente. Sobresale el desarrollo del currículo oculto que los profesores sustentan en desmedro de la filosofía de la universidad.

Un aporte, para el reenfoque hacia la formación docente en las universidades pedagógicas, podría venir de su epistemología. Epistemología puede entenderse “como fundamento” en las ciencias. Si es fundamento, entonces es un equivalente a una relación causal donde "los sujetos se representan a sí mismos y al mundo, del sentido de ser y de los modos de realizarlos” (Vargas Guillén, 2003, p. 6); esto se da porque la epistemología "se ocupa de los fundamentos y procedimientos de todas las ciencias” (Bunge, 2002, p. 138) y permite una clara relación con la teoría del conocimiento, que es precisamente el ámbito de este ensayo. Por tanto, se asume que es en las universidades pedagógicas que se dedican a la construcción de conocimientos y la formación de conceptos, para la formación docente que es el espacio adecuado, para promover los cambios que se requieren en la educación nacional.

\section{Las ciencias y las universidades pedagógicas}

Las ciencias solamente pueden enseñarse en las universidades donde se garantiza el desarrollo integrado de la investigación con la docencia y con la extensión, para vincular sus resultados con la solución de los problemas de la sociedad, incluyendo los educativos. Es en esas aulas donde el profesor sabe que debe proponer acciones didácticas que hagan que los saberes sabios sean 
transformados en saberes a enseñar, para garantizar que los estudiantes aprendan a formar conceptos y a construir conocimientos que sirvan a las exigencias de la sociedad. Acciones que muestren a los profesores asumiendo una actitud científica en su vida como profesionales liberales o de la educación.

Las ciencias están asociadas con "algo que tenemos que descubrir y no algo que podamos establecer de antemano mediante un argumento filosófico” (Chalmers, 1988, p. 229). Quiere decir que el descubrir es el camino a las ciencias y los argumentos filosóficos pueden ayudar a determinar su esencia. Se acepta que la ciencia se caracteriza como un "conocimiento racional, sistemático, exacto, verificable y por consiguiente falible” (Bunge, 2001, p. 11),por tanto, se posibilitan las miradas paradigmáticas en su desarrollo. Khun (1978, p. 13) define a los paradigmas "como realizaciones científicas universalmente reconocidas que, durante cierto tiempo, proporcionan modelos de problemas y soluciones a una comunidad científica”. Miradas que posicionan a los científicos en la búsqueda de nuevos conocimientos que pueden ayudar a mejorar las condiciones de vida de la humanidad. Esto es, búsqueda de la esencia: la formación docente.

En la discusión sobre la epistemología de la formación docente, en adelante se abordarán otras intenciones epistemológicas que le den fuerza al ensayo, tales como: epistemología de la educación, de la investigación y de la formación de docentes.

\section{Epistemología de la Educación}

Las ciencias de la educación se desarrollan inmersas en discursos que obedecen a las ciencias que las conforman como: pedagogía, didáctica, andragogía, psicología, sociología, antropología, etc. Cada ciencia condiciona una forma de expresar los conceptos asociados a la enseñanza y al aprendizaje. Estos discursos son los que hacen ver al profesor como un repetidor inconsciente de conceptos, pero convencido de una práctica pedagógica especial.

Una posibilidad de un discurso propio de las ciencias de la educación está relacionada con la acción comunicativa presente en el acto de enseñar y de aprender en las aulas. Este hecho, por sí mismo, es el que debe posicionar al docente en la perspectiva de enseñar desde el aprender. ¿Qué significa enseñar desde el aprender? En principio, que el docente se ubique en la posición del que aprende siempre, para que en el camino al aprender sienta que está en capacidad de enseñar, por eso 
el discurso de las ciencias de la educación debería ser el de la pedagogía. Sobre todo, porque la comunicación "entre maestro y alumno es [una comunicación activa], de relaciones recíprocas, por lo que todo maestro sigue siendo alumno y todo alumno es maestro” (Gramsci, 2001, p. 47).

Ello supone que la formación humana es la condición que prepara a las personas para actuar en una sociedad con determinadas perspectivas: afectivas, prácticas, teóricas, que es lo que define que un profesional dedicado a la enseñanza deba enseñar con la convicción del conocimiento que adquiere en su aprendibilidad, enseñabilidad y educabilidad que se refleja en un aprender a enseñar, para enseñar a aprender de manera comprometida.

Siguiendo a Gallego Badillo y Pérez Miranda (1999, p. 95) la aprendibilidad es "una cualidad que le confiere cada estudiante a ese saber para hacerlo objeto de aprendizaje”. Esto requiere "que el profesor admita de entrada que las mentes de los alumnos no están vacías y que, por tanto, poseen explicaciones acerca de los fenómenos de los que da cuenta la ciencia que él enseña”.El aprendiente está consciente que su aprendizaje depende de él mismo en relación con los aportes de sus profesores. Quienes "realizan su trabajo en el interior de su compromiso con un proyecto epistemológico, pedagógico y didáctico, de carácter investigativo, teóricamente fundamentado” (Gallego Badillo y Pérez Miranda, 1999, p. 100) propio, que es a lo que se llama enseñabilidad. Lo que exige que los enseñantes sean profesores que expliciten el acto de enseñar a aprender en cada momento de sus realizaciones como docente.

Las relaciones entre aprendibilidad y enseñabilidad garantizan que "las lógicas de las intencionalidades curriculares, en la dirección incuestionable de que todo educando ha de, finalmente, incorporarse críticamente al proyecto cultural, social, político y económico y a sus procesos de producción de bienes y servicios, con miras a sostener financieramente su proyecto ético de vida” (Gallego Badillo y Pérez Miranda, 1999, p. 111),que es lo que le da cuerpo a la educabilidad. Es el momento en que se concreta el enseñar desde el aprender y lo vuelve competente, para enseñar a aprender toda su vida. Esta conexión de formación es la posibilidad que da la pedagogía, para orientar el discurso de las ciencias de la educación. 


\section{Epistemología de la investigación}

La construcción de conocimientos es el saber hacer de la epistemología de la investigación por eso se puede "afirmar que la epistemología del ser, bajo sus diversas articulaciones, no es el método de conocer el ser; sino de hacerse episteme el ser” (Vargas Guillén, 2005, p. 82).

Esto implica la producción de conocimientos en una perspectiva objetiva/subjetiva porque como dice Habermas (2002, p. 233): "El conocimiento no se produce ya en la correspondencia de las oraciones con los hechos", pues "la objetividad queda asegurada cuando el sujeto representador se refiere de manera correcta a su objeto", para hacer el cambio que se logra "mediante una coincidencia pública, alcanzada en la comunidad de comunicación...”, que es lo que implica llegar al consenso entre los humanos y de esa manera establecer verdades plausibles. Se trata de ajustar las teorías a las prácticas en el descubrimiento de la verdad.

\section{Epistemología de la formación de docentes}

Las universidades que se dedican a la formación de docentes lo hacen según expectativas y potencialidades de sus políticas, objetivos, modelo, enfoque, currículo y mecanismos académicoadministrativos, propios de la formación perfilada. Estos elementos son los determinantes en la precisión del tipo de formación docente. A continuación, se discuten los conceptos que ayudan a interpretar mejor el sentido de la epistemología de la formación de docentes.

Modelo Educativo: El modelo educativo en una universidad es la teoría a probar en la formación de un profesional con características propias del acto formador preestablecido desde lo pedagógico-académico, dirigido con una perspectiva teórico-metodológica atinente a un currículo particular, lo que lo liga a un modelo científico. Un modelo científico es "una representación provisoria, perfectible e idealizada de una entidad o fenómeno físico” (Bunge, 1985) citado en Raviolo, Ramírez y López(2010, p. 591) que se expresa en una "representación simplificada de un hecho, objeto, fenómeno, proceso, realizada con la finalidad de describir, explicar y predecir”. Se 
trata, entonces, de una construcción humana en la que se busca “conocer, investigar y comunicar” pensamientos, acciones y emociones.

Con propósitos de ejemplo se hace una revisión de los modelos educativos de la UNAH y de la UPNFM. Ambas universidades definen sus expectativas de formación de profesionales de diferentes áreas (la primera) y de docentes (la segunda) que suponen una teoría en el modelo educativo declarado. La UNAH (2008, pp. 23-30) considera que "el modelo educativo es la forma totalizadora en que una comunidad educativa histórica y culturalmente situada, siente, piensa, organiza su quehacer haciendo realidad el hecho educativo como tal”. Esto permite a sus autoridades y docentes visualizar un tipo de formación profesional como abogado, médico, pedagogo.

En cambio, la UPNFM (2014, pp. 30-33) asume que: “Un modelo educativo consiste en una recopilación o síntesis de distintas teorías y enfoques pedagógicos que orientan a los docentes en la elaboración de los planes de estudios y en la sistematización del proceso educativo...”, esto indica que el modelo de la UPNFM es un patrón conceptual, para el tipo de docente requerido en el país.

En ambas universidades se defiende una postura que le es propia del modelo educativo que estima un tipo de formación de un profesional con un discurso que viene de la pedagogía crítica. En la UNAH "se construyó tomando como base la teoría constructivista, la teoría crítica y la teoría humanista” (p. 34). En la UPNFM se construyó como un “un proceso crítico-constructivo” (p. 22). Perspectiva que argumenta a favor de mecanismos académicos y administrativos estrictos en la formación de un profesional crítico y reflexivo. En la UNAH está por verse la influencia de este enfoque hasta en la formación de pedagogos y en la UPNFM que asumió la formación basada en competencias en el 2008, distinto de la idea de la pedagogía crítica, aún no se conocen resultados específicos del modelo.

Por el condicionamiento que provocan ambos enfoques, en la literatura existe una sistematización de los modelos teóricos en la formación de docentes: "práctico artesanal, academicista, tecnicista-eficientista y hermenéutico-reflexivo” (De Lella, 1999). En el modelo 
práctico artesanal debe formarse una persona que toma la docencia como un oficio, independientemente de sus concepciones sobre la enseñanza y las prácticas pedagógicas. El segundo considera a la docencia con alto sentido de responsabilidad teórica y un dominio de los conceptos y elementos que le dan vida a una forma de expresar la formación docente. El tercero se asocia a un profesional que busca garantizar la eficiencia en función de la técnica con la incorporación de las nuevas tecnologías de la información y la comunicación como la panacea. Y el cuarto, supone que la profesión docente está inscrita en la complejidad de los actos de enseñanza por lo complejo de las acciones pedagógico-didáctico-psicológicas propias del acto de enseñar.

El docente formado, según el modelo hermenéutico-reflexivo, debería observar un conocimiento experto, como el mejor en su campo, con concepciones sobre la enseñanza y las prácticas pedagógicas atinentes y propias del saber enseñar. Concepciones y prácticas pedagógicas que determinan un estilo de enseñanza o como práctica o como conocimiento o como pensamiento (Martínez, 2003). La búsqueda es la enseñanza como pensamiento y una práctica pedagógica que integre la teoría con la práctica.

La idea es que la formación docente rompa con las tradiciones tradicionalistas y vaya en pos de una tradición crítica, reflexiva y proactiva, donde los formadores sean los provocadores o desafiadores de un nuevo tipo de docente. Conviene ir más allá de lo negro o blanco en las posiciones en la enseñanza y el aprendizaje. Eso es buscar los grises que están en las combinaciones de la formación de conceptos y la construcción de conocimientos en la formación docente. El reto es desarrollar incertidumbres, dudas, dilemas, no verdades absolutas, para la formación docente de los nuevos profesores, independientemente de los enfoques.

Enfoque: Cuando se habla de enfoque se condicionan las enseñanzas o los aprendizajes a una forma de ver el mundo, a una perspectiva particular de hacer observable las conductas de las personas en situaciones particulares o específicas de la vida. El conductismo es un enfoque que desarrolla el concepto estímulos-respuesta. El constructivismo apuesta por la consideración de los conocimientos previos de los aprendientes para dar el salto a otro estadio de conocimiento. Y la formación basada en competencias que implica el logro de eficiencia y eficacia (venida de la industria y el comercio), para la formación de un profesional exitoso. 
Es el momento de aceptar que, desde la psicología, la educación se ha movido del conductismo, al constructivismo y ahora a la formación basada en competencias. Cada una de ellas con características propias y que fortalecen un tipo de formación de docentes, no necesariamente una sólida formación docente. La psicología conductista supone el empleo de procedimientos, para experimentar el estudio del comportamiento humano observable, que no es más que hacer visible el concepto estímulo-respuesta.

Con el constructivismo las condiciones se amplían en el sentido de facilitar espacios de construcción del conocimiento. Por eso es entendible que: "El sujeto construye el conocimiento de la realidad, ya que ésta no puede ser conocida en sí misma, sino a través de los mecanismos cognitivos de que se dispone, mecanismos que, a su vez, permiten transformaciones de esa misma realidad” (Araya, Alfaro y Andonegui, 2007, p. 77).

Una nueva circunstancia se presenta cuando se habla del enfoque basado en competencias. Lo visible es que hay acuerdos y desacuerdos entre los investigadores sobre su uso. Los primeros asumen las directrices sin discusión y los segundos piensan que deben ir más allá de las competencias como es el caso de Barnett (2001, p. 249) que las llama ideologías y propone argumentos que trascienden al mundo de la vida. Epistemológicamente hablando, el saber cómo responde a una lógica operatoria, el saber qué al dominio del contenido, y más allá de las competencias está el mundo de la vida del profesor desarrollando propuestas de construcción de conocimientos de manera reflexiva, crítica y proactiva. Significa que, para la formación docente en el enfoque basado en competencias, habría que ir más allá de las competencias.

El currículum: El currículo es un proyecto de construcción cultural (Grundy, 1998) que define los alcances de la formación profesional de las personas que estudian en una universidad. Se considera que el currículo es un concepto reciente en las universidades, por eso la práctica del currículum está centrada en "comportamientos didácticos, políticos, administrativos, económicos, etc., detrás de los que se encubren muchos supuestos, teorías parciales, esquemas de racionalidad, creencias, valores, etc.” (Sacristán, 1998, p. 13). 
Esto sugiere que enseñar para la vida supone la presencia de un profesor concentrado en la investigación (Stenhouse, 1998), para cambiar la imagen profesional de un profesor. Significa que el currículo debe garantizar el tipo de docente a formar, para acercar ese profesional a las exigencias de la sociedad, para mejorar la calidad de la enseñanza, del aprendizaje, de la formación docente, de la educación.

\section{Epistemología de la formación docente}

La formación docente es la esperanza en el devenir de la educación de los pueblos. ¿Qué implica la formación docente? Una aventura epistemológica que está relacionada con la formación de un profesional competente por la capacidad de interpretar la realidad de los educandos y ponerla en perspectiva de formación hasta garantizar una acción profesional con conocimiento y práctica orientada a la solución de problemas de la vida diaria.

“Se trata de ver la formación como un aprendizaje constante, acercando ésta al desarrollo de actividades profesionales y a la práctica profesional y desde ella” (Imbernón, 2007, p. 11). Esto es lo que se aprende de la visión de Gorodokin (2000, p. 2) citando a Achilli (2000) cuando afirma que "la formación docente puede comprenderse como un proceso en el que se articulan prácticas de enseñanza y de aprendizaje orientadas a la configuración de sujetos docentes/enseñantes. La práctica docente se concibe en un doble sentido: como práctica de enseñanza, propia de cualquier proceso formativo y como apropiación del oficio de docente, cómo iniciarse, perfeccionarse y/o actualizarse en la práctica de enseñar”.

La formación docente, en consecuencia, se concentra en la conformación de un profesional que se dedicará a ejercer la docencia toda la vida. La expectativa es que cuando una persona ha sido formada para enseñar y se mantiene en el medio, es porque está convencido que la docencia será su ámbito laboral y por el cual luchará hasta lograr que otras personas aprendan y se comprometan consigo mismo. Ello, implica reconocer que se ha logrado el objetivo: saber enseñar desde el aprender. Dar este paso es reconocer que la formación docente implica un trabajo metódico y sistemático en función de concepciones sobre la enseñanza y prácticas pedagógicas atinentes con el pensamiento contemporáneo. 
Concepciones sobre la enseñanza: A lo largo del tiempo ha habido diferentes concepciones sobre la enseñanza y eso ha determinado cómo enseñar en las aulas, considerando que, “dentro del aula y fuera de ella, subyacen concepciones pedagógicas, [que] describen y explican los fenómenos educativos que se dan tanto en su estructura como en su funcionamiento...” (Beltrán y otros, 2008, p. 42). Esto implica considerar a "la enseñanza como posibilidad de pensamiento” (Martínez, 2003) estricto. Cada reflexión o acción en la enseñanza obliga estar en el acontecimiento (lo que trasciende) y no sólo en lo que acontece (lo evidente en las aulas), pues "el acontecimiento no se queda allí, pasa por allí, pero no se agota” (Martínez, 200, p. 207).

En estas circunstancias, "la enseñanza pasa por el lenguaje, pasa por unos sujetos, [y por tanto es] una categoría que permitiría caracterizar conocimientos, sujetos y prácticas” (Martínez, 2003, p. 208). Por tanto, debe entenderse que "los profesores son producto de un largo proceso de formación y socialización, durante el cual fueron integrando supuestos, creencias, teorías implícitas y explícitas” (Sanjurjo, 2002, p. 25) que le dan sentido y significado propio a sus enseñanzas, lo que condiciona una enseñanza como pensamiento. Esto implica el desarrollo de prácticas pedagógicas contextualizadas.

Prácticas Pedagógicas Contextualizadas: La práctica es entendida como un campo de aplicación de la teoría. Se podría pensar que sin teoría no hay práctica. En la enseñanza y sobre todo en la formación docente tiene validez esa misma visión, pues se encarna en "el conocimiento y la acción, la acción es tan sólo una implementación y una comprobación de la decisión técnica” (Schön, 1992, p. 82).

Si las prácticas pedagógicas son un conjunto de estrategias e instrumentos (herramientas) que debería utilizar el profesor para ayudarles a sus estudiantes en el proceso de aprender a enseñar (Marcelo, 2001) para enseñar a aprender (Bixio, 2002), estarían mostrando que su responsabilidad sería establecer con precisión su propia filosofía de enseñanza en el marco de la filosofía y políticas institucionales, que es lo que determinaría la formación docente. Entonces, el saber enseñar pasa por aprender a enseñar para enseñar a aprender toda una vida. Lo que exige el desarrollo sistemático de 
métodos, técnicas, estrategias y procesos en el acto de enseñar, independientemente del tipo de profesional a formar.

Métodos, técnicas, estrategias y procesos de enseñanza: Todos, profesores y estudiantes, en o afuera de las aulas, hablan de métodos, técnicas, estrategias y procesos como lo sabido. Pero cuando se les confronta, sobre ellos, lo hacen con distinto sentido y significado; lo apropiado sería comunicarse con los mismos códigos lingüísticos para no romper con la comunicación verdadera que debe haber entre los profesionales de la educación.

Métodos: Los métodos son orientadores de procesos que ayudan a perfilar la formación de conceptos y la construcción de conocimientos por parte de los profesores y estudiantes. Son el sentido de una práctica pedagógica contextualizada. Siguiendo a Nérici (1985, pp. 35-40) “método quiere decir camino para alcanzar los objetivos estipulados en un plan de enseñanza”. Es la realización de una actividad que sigue un camino en espiral, para generar aprendizajes. Se incorpora el método diálogo reflexivo (Umanzor, 2011) para fortalecer la idea de camino y proceso a la vez.

El diálogo reflexivo "permite el aprendizaje críticamente reflexivo, [porque] compromete a la persona hasta el extremo de sus conocimientos, su sentido del yo y del mundo, tal como lo experimentamos. Por tanto, se cuestionan nuestras premisas vigentes sobre el saber, sobre nosotros mismos y nuestro mundo” (Brockbank y McGill, 2002, p. 73).Esta dimensión se asocia con el método diálogo reflexivo, así:

Reflexión desde la práctica.La idea es que yo como profesor o profesora me mantenga “reflexionando sobre mis enfoques respecto a mi forma de enseñar y de los fines que persigo con mi enseñanza” (Brockbank y McGill, 2002, p. 89)”.Implica la realización de un diagnóstico en dos niveles: los aportes del docente y las experiencias de los estudiantes, para favorecer la continuidad del proceso enseñanza-aprendizaje. Se trata de establecer las condiciones básicas de entrada, para replantear la nueva situación didáctica. En la UPNFM existe una buena cantidad de estudiantes que trabajan en la docencia y han acumulado experiencias ricas que deberían ser la base de su formación 
docente, lo que invita a acciones didácticas e investigativas propias, para aprender a enseñar y luego enseñar a aprender.

Reflexión teórica. Es la posibilidad de fundamentar teóricamente una acción didáctica con conocimiento, para establecer que el "saber qué" está por sobre el "saber cómo”. Esto es, aprender conceptos, principios, normas, leyes y sus aplicaciones en el marco de la ciencia particular y la didáctica, que es lo que garantizará el plus académico. Se trata de poner en discusión las teorías que sostienen las concepciones sobre la enseñanza y las prácticas pedagógicas contextualizadas.

Acción reflexiva.La acción reflexiva se da cuando "se cuestionan las premisas vigentes sobre las ideas, el yo y el mundo. Ese aprendizaje se transforma en reflexivamente crítico cuando las ideas emergentes se relacionan con los sentidos que se le dan al saber, al yo y al mundo, y surge una nueva comprensión” (Brockbank y McGill, 2002, p. 73). Es el momento de la relación de los conocimientos previos de los estudiantes con los aportes de las teorías actuales respecto del enseñar a aprender, para fortalecer una práctica reflexiva propia del saber enseñar y del ser docente.

Reflexión en la acción.La reflexión en la acción se da cuando el docente "piensa sobre la marcha”. Es decir, que la reflexión en la acción "se produce cuando nos encontramos en medio de una acción y, al hacerla mostrarnos reflexivos en la acción” (Brockbank y McGill, 2002, p. 90)lo que provoca sorpresa, que es lo que determina la nueva acción. Esto significa que la reflexión en la acción tiene un referente inmediato que es la acción y parte de un pensamiento que influye en lo que se hace como la condición sabida: ser profesor que enseñará a aprender toda una vida.Estos pasos en espiral están mediados por preguntas-problematizadoras que desafían la consciencia de las personas, para estar en la acción.

Técnicas: En cuanto a las técnicas, es conocido que existe una variedad de ellas y que tienen un soporte psico-pedagógico-didáctico propio. Muchas de ellas fijan una posición del coordinador de las mismas (el profesor) y de los actores (los estudiantes). El uso de estas técnicas está relacionado con la necesidad de profundizar acciones que fundamenten la formación de conceptos y la construcción de conocimientos de manera expresa. 
Siguiendo a Nérici (1985) “técnica significa cómo hacer algo”. Esto es, la técnica precisa de eventos secuenciales, pero en su desarrollo íntimo responden a una situación particular. Existen técnicas para presentación, para análisis, para reflexión; y cada una es distinta y se aplica en una parte del desarrollo del método. La técnica no sustituye al método, pero juntos deben llevar al educando a observar, criticar, juzgar, sacar conclusiones, correlacionar, diferenciar, sintetizar, conceptualizar y reflexionar, que es a lo que se aspira con la teoría enseñar desde el aprender.

Estrategias: Siguiendo a Zapata (2003, p. 8) "las estrategias de enseñanza se conciben como los procedimientos utilizados por el docente para promover aprendizajes significativos, implican actividades conscientes y orientadas a un fin”. Según esta definición, las estrategias son sinónimos de procedimientos, entendiendo que procedimiento es una acción no secuente que puede ayudar a lograr un fin, entonces, la estrategia tiende a ser un evento, también, sobresaliente, único y de desarrollo continuo para avivar y activar el aprendizaje de los estudiantes. La presentación de una lámina es una estrategia plausible para iniciar la enseñanza de un concepto, tema. La forma que se utilice es la que garantizará el aprendizaje de los estudiantes.

Procesos: Los profesores también hablan de procesos, pero existen diferencias sustantivas en la teoría y práctica de los mismos. Aunque en la enseñanza se habla más de método, aquí se le llama proceso: inducción, deducción y también, analogía, con la idea de apostar por el desarrollo del razonamiento lógico o pensamiento lógico de las personas. Siguiendo a Alfonso Sánchez (2003,s.p.): “El proceso de enseñanza, con todos sus componentes asociados, debe considerarse como un sistema estrechamente vinculado con la actividad práctica del hombre, que, en definitiva, condiciona sus posibilidades de conocer, comprender y transformar la realidad que lo circunda...”.

Por eso, se asume que el proceso inductivo es una secuencia de eventos que se suceden uno a uno y que dejan en el camino un aprendizaje significativo, así: 1. Presentación de un problema. Referido a una oportunidad de empezar desde la práctica. 2. Comprensión del problema. Se trata de mostrar la interpretación que una persona le da al problema. 3. Identificación de estrategias o procedimientos que resuelvan el problema, implica pensar en una oportunidad de actuación. 4. Resolución del problema. Es la confirmación de que la estrategia elegida es la correcta y posibilita avanzar. 5. Verificación de la solución del problema. Se trata de confirmar que los resultados son 
los correctos y adecuados. 6. Definición del concepto expresado en el problema. Se trata de que el estudiante llegue a expresar el desarrollo de su pensamiento lógico.

El proceso deductivo, en cambio, es visto como una relación inversa pero no necesariamente; se trata de partir de una visión genérica y establecer los mecanismos que le permitan al estudiante llegar a un resultado final común y especial para todos, así: 1. Presentación del tema y su objetivo. 2. Definición del tema, para su análisis. 3. Comprensión de la definición, para saber qué hacer. 4. Presentación de un problema-ejemplo para establecer sus aplicaciones. 5. Resolución del problema y su revisión. 6. Reconstrucción de la definición, para reafirmar la definición inicial.

En el proceso por analogía, en cambio, lo que se busca es la comparación constante de eventos similares entre teorías o prácticas relacionadas con la resolución de problemas de una manera diferente, como una simbiosis necesaria para que se convierta en una nueva opción metodológica en la solución de un problema, así: 1. Presentación de un problema o tema, para aprender a leer comprensivamente. 2. Análisis de los conceptos específicos del problema. 3. Revisión de las opciones de resolución del problema, para establecer su comprensión y razonamiento. 4. Resolución del problema y revisión de sus resultados. 5. Comparación de los conceptos de manera que resalten las semejanzas o las diferencias, para asumir el concepto como la verdad.

Los tres procesos: inductivo, deductivo y analógico son tres diferentes formas de acercarse a la solución de un problema como la esencia del desarrollo del pensamiento lógico de las personas. En resumen, las concepciones sobre la enseñanza y las prácticas pedagógicas, los métodos, técnicas, estrategias y procesos son la esencia del desarrollo de las prácticas pedagógicas contextualizadas. Lo anterior supone que la epistemología de la formación docente está asociada a la carga teóricometodológica desarrollada en los procesos de formación de docentes en las universidades dedicadas a este propósito. 
Si en la formación de distintos profesionales hay confusión en los conceptos formadores, quedan en el ambiente una serie de preguntas: ¿Cómo el abogado, que es profesor, transfiere su saber al nuevo abogado que forma? ¿Cómo el médico, que es profesor, transfiere de su saber al nuevo médico? Y ¿cómo el profesor, formador de profesores, forma a los nuevos profesores? En todos los casos, la formación de ese profesional o docente no se hace por ósmosis. ¿Qué lo garantizaría, realmente?

\section{A MANERA DE CONCLUSIONES}

El desarrollo de las ciencias es la aspiración de los científicos y los espacios para ser enseñadas son las aulas de las universidades que apuestan por la formación de un profesor-investigador. Esto sugiere la presencia de profesores formadores investidos de conceptos, procesos y teorías, para nutrir a las ciencias, pero más para la formación docente de los profesores del futuro. La epistemología de la educación supone el manejo de discursos y realizaciones que deben conducir la formación humana y, sobre todo, la de los docentes que saben enseñar desde el aprender. Esta circunstancia, por sí mismo, consustancia los procesos de enseñanza-aprendizaje, para la formación de conceptos y la construcción de conocimientos que da lugar a la producción científica y a la formación de profesores críticos, reflexivos y proactivos.

La formación docente en las universidades pedagógicas debe pasar por la precisión de mecanismos académicos propios del enseñar desde el aprender, para el mundo de la vida. Así, el docente, formado como tal, estaría en condiciones de un manejo estricto de los conocimientos producidos en una ciencia determinada con los enlaces específicos a las otras ciencias y sobre todo aquellos centrados en lo pedagógico-didáctico-psicológico, para saber enseñar. El saber enseñar es la clave en todo el proceso formador, por tanto, desde las ciencias de la educación se deben garantizar el modelo, el enfoque, el currículum, los planes de estudio propios de la circunstancia en la formación docente.

Se trata de asegurar que la formación docente sea el objetivo, la clave en los procesos formativos, con universidades pedagógicas que establezcan los mecanismos académicoadministrativos propios de la formación pretendida y no divagar en discursos cargados de eufemismos ortodoxos que no contribuyen al logro de los objetivos formadores de los docentes que requiere un país como Honduras: docentes que saben enseñar desde el aprender. 


\section{REFERENCIAS BIBLIOGRÁFICAS}

Alfonso Sánchez, Ileana (2003). Elementos conceptuales básicos del proceso de enseñanzaaprendizaje. Red Telemática de Salud en Cuba (Infomed). Recuperado dehttp://bvs.sld.cu/revistas/aci/vol11_6_03/aci17603.htm

Araya, Valeria; Alfaro, Manuela; Andonegui, Martín (2007). Constructivismo: Orígenes y Perspectivas. $\quad$ Laurus, 13(24), 76-92. Recuperado de http://redalyc.uaemex.mx/src/inicio/ArtPdfRed.jsp?iCve=76111485004

Barnett, Ronald (2001). Los límites de la competencia: El conocimiento, la educación superior y la sociedad. España: Primera edición, Editorial Gedisa, Traducción de Adelaida Ruiz.

Beltrán Villamizar, Yolima, Quijano Hernández, Martha Helena y Villamizar Acevedo, Gustavo Alfonso (2008). Concepciones y prácticas pedagógicas de los profesores que enseñan ciencias naturales y ciencias humanas en programas de ingeniería de dos universidades colombianas. $\begin{array}{lll}\text { STUDIOSITAS, 3(1), } & \text { Recuperado }\end{array}$ dehttp://portalweb.ucatolica.edu.co/easyWeb2/files/21_986_v-3-n-1-i-beltran-y-otros.pdf

Bixio, Cecilia (2002). Enseñar a aprender. Construir un espacio colectivo de enseñanzaaprendizaje. Argentina: $3^{\mathrm{a}}$. Ed. Ediciones Homo Sapiens, Serie: Educación.

Bunge, Mario (s.f.). La ciencia. Su método y su filosofía. Recuperado dehttp://users.dcc.uchile.cl/ cgutierr/cursos/INV/bunge_ciencia.pdf

Brockbank, Anne y McGill, Ian (2002). Aprendizaje reflexivo en la educación superior. Madrid: Traducción de Pablo Manzano, Ediciones Morata.

Chalmers, Allan (2012). ¿Qué es esa cosa llamada ciencia? México: Tercera edición, siglo veintiuno editores. Recuperado dehttps://ulagos.files.wordpress.com/2012/03/libro-que-es-esa-cosallamada-ciencia.pdf

De Lella, Cayetano (1999). Modelos y tendencias de la Formación Docente. I Seminario Taller sobre Perfil del Docente y Estrategias de Formación. Recuperado dehttp://www.oei.es/cayetano.htm Gallego Badillo, Rómulo y Pérez Miranda, Roymán (1999). Aprendibilidad, Enseñabilidad y Educabilidad en las Ciencias Experimentales. REVISTA EDUCACIÓN Y PEDAGOGÍA XI(25), 
89-117.

Recuperado

dehttp://aprendeenlinea.udea.edu.co/revistas/index.php/revistaeyp/article/viewFile/5861/5274

Gorodokin, Ida C. (s.f.). La formación docente y su relación con la epistemología. Instituto de

Formación Docente Continua San Luis. Recuperado de

http://rieoei.org/deloslectores/1164Gorodokin.pdf

Gramsci, Antonio (2001). La Alternativa Pedagógica. México: Sexta edición, Editorial Fontamara, 47.

Grundy, Shirley (1998). Producto o praxis del currículum. Madrid: Tercera edición, Editorial Morata, Traducción de Pablo Manzano, Colección: Pedagogía. La pedagogía hoy.

Habermas, Jürgen (2002). Teoría de la acción comunicativa, I. Racionalidad de la acción y racionalización social. España: Editorial Taurus Humanidades, Traducción de Manuel Jiménez Redondo, Versión Castellana.

Imbernón, Francisco (2007). La Formación y el desarrollo profesional del profesorado. Hacia una nueva cultura profesional. España: $7^{a}$. Edición, Editorial Graó.

Kuhn, Thomas S. (1978). La estructura de las revoluciones científicas. México: Tercera reimpresión, Fondo de Cultura Económica, Traducción de Agustín Contín.

Martínez, Alberto (2003). La enseñanza como posibilidad de pensamiento. Colombia: En Zuluaga y otros (2003). Pedagogía y Epistemología, Colección: Pedagogía e historia, Cooperativa editorial Magisterio, Grupo Historia de la Práctica Pedagógica.

Marcelo, Carlos (2001). Aprender a enseñar para la Sociedad del Conocimiento. En Revista Complutense de Educación, 12(2), 531-593. Recuperado de http://www.ucm.es/BUCM/revistas/edu/11302496/articulos/RCED0101220531A.PDF

Morin, Edgar(s.f.). Introducción al pensamiento complejo. Recuperado de http://www.pensamientocomplejo.com.ar/docs/files/MorinEdgar_Introduccion-al-pensamientocomplejo Parte1.pdf

Nérici G., Imídeo (1985). Metodología de la enseñanza. México: Editorial Kapelusz, Colección: Actualización Pedagógica. 
Raviolo, Andrés; Ramírez, Paula y López, Eduardo A. (2010). Enseñanza y aprendizaje del concepto de modelo científico a través de analogías. En Rev. Eureka Enseñ. Divul. Cien., 7(3), 581-612. Recuperado de http://reuredc.uca.es/index.php/tavira/article/viewFile/66/59

Sacristán, J. Gimeno (1998). El currículum: una reflexión sobre la práctica. Madrid (España): $7^{a}$. ed., Ediciones Morata, Colección: Pedagogía Manuales.

Sanjurjo, Liliana (2002). La formación práctica de los docentes. Reflexión y acción en el aula. Argentina: Homo Sapiens Ediciones.

Schön, Donald A. (1992). La formación de profesionales reflexivos. Hacia un nuevo diseño de la enseñanza y el aprendizaje en las profesiones. Madrid: Editorial Paidós, Temas de Educación, Ministerio de Educación y Ciencia, Traducción de Lourdes Montero y José Manuel Vez Jeremías.

Stenhouse, Lawrence (1998). Investigación y desarrollo del currículum. España: 4a. ed., Ediciones Morata, Colección: PEDAGOGÍA. La pedagogía hoy.

Umanzor, Pastor (2011). Construcción de un diálogo reflexivo a partir de concepciones sobre la enseñanza y prácticas pedagógicas. Tegucigalpa: Tesis doctoral.

UNAH (2008). Modelo Educativo. Tegucigalpa: Editorial Universitaria.

UPNFM (2014). Modelo Educativo. Tegucigalpa: Editorial Universitaria.

Vargas Guillén, Germán (2003). Tratado de Epistemología. Fenomenología de la ciencia, la tecnología y la investigación social. Colombia: Editorial San Pablo. Serie: Filosofía.

Vargas Guillén, Germán (2005). La experiencia de ser. Tratado de Metafísica. Colombia: Editorial San Pablo.

Zapata, V. (2003). Manual de estrategias de enseñanza/aprendizaje. Servicio Nacionalde Aprendizaje, Centro Metalmecánico. Recuperado de http://www.cepefsena.org/documentos/METODOLOGIAS\%20ACTIVAS.pdf 